\title{
NEDD4 E3 ubiquitin protein ligase serves an important role in cutaneous melanoma occurrence and development
}

\author{
FANG CHENG $^{1}$, YI CHENG ${ }^{2}$, XIAOLING ZHAO ${ }^{1}$, LIHUI AN ${ }^{1}$, \\ LINFANG YANG $^{1}$, ZIHAN LI ${ }^{1}$, LEI ZHANG ${ }^{3}$ and RUNZHI HE ${ }^{4}$ \\ ${ }^{1}$ Department of Dermatology, Affiliated Xingtai People's Hospital of Hebei Medical University, Xingtai, Hebei 054001; \\ ${ }^{2}$ Department of Dermatology, The Fourth Hospital of Hebei Medical University, Shijiazhuang, Hebei 050000; \\ ${ }^{3}$ Department of Dermatology, The Second People's Hospital of Guiyang, Guiyang, Guizhou 550023; \\ ${ }^{4}$ Department of Neurosurgery, Affiliated Xingtai People's Hospital of Hebei \\ Medical University, Xingtai, Hebei 054001, P.R. China
}

Received March 19, 2021; Accepted June 25, 2021

DOI: $10.3892 /$ etm.2021.10818

\begin{abstract}
The present study aimed to discuss the effects and relative mechanisms of NEDD4 E3 ubiquitin protein ligase (NEDD4) in cutaneous melanoma (CMM) occurrence and development. Clinical cancer and adjacent normal tissues samples were collected to analyze pathological changes and protein expression of NEDD4. Moreover, small interfering (si) RNA was used to knockdown NEDD4 expression in SK-MEL-2 and Malme-3M cells. Cellular proliferation, apoptosis, invasiveness and migration were examined using colony formation, flow cytometric, Transwell and wound-healing assays, respectively. In addition, the relative mRNA and protein expression levels of NEDD4, notch receptor 1 (Notch1) and PTEN were evaluated via reverse transcription-quantitative (RT-q) PCR and western blotting. It was found that NEDD4 mRNA and protein expression were significantly upregulated (both $\mathrm{P}<0.01$ ). Following NEDD4-knockdown, colony number was significantly decreased, while the apoptotic rate was significantly increased, the invasive cell number was significantly inhibited and the wound-healing capacity was significantly decreased. Following si-NEDD4 transfection, RT-qPCR and western blotting revealed that NEDD4 and Notch1 mRNA and protein expression levels were significantly downregulated, while those of PTEN were significantly upregulated in the SK-MEL-2 and Malme-3M
\end{abstract}

Correspondence to: Dr Lei Zhang, Department of Dermatology, The Second People's Hospital of Guiyang, 547 Jinyang South Road, Guanshan Lake District, Guiyang, Guizhou 550023, P.R. China E-mail: maomingfeng0219@163.com

Dr Runzhi He, Department of Neurosurgery, Affiliated Xingtai People's Hospital of Hebei Medical University, 16 Hongxing Street, Xingtai, Hebei 054001, P.R. China

E-mail: herunzhi0213@163.com

Key words: NEDD4 E3 ubiquitin protein ligase, cutaneous melanoma, SK-MEL-2, malme-3M, notch receptor 1, PTEN cell lines. Collectively, the current results suggest that NEDD4-knockdown effectively suppressed CMM biological activity by regulating the Notch1/PTEN pathway in vitro.

\section{Introduction}

Immunotherapy has become one of the most rapidly developed treatment methods in the field of cancer, which essentially depends on activating the patient's own immune system against tumors (1). A tumor vaccine is used to introduce various forms of tumor antigen, which can induce cellular and humoral immunity, as well as generating long-term immune memory, thereby controlling or preventing tumor progression (2).

Melanoma is a highly malignant tumor type, prone to invasion and metastasis, with a poor prognosis (3). Melanoma also has strong immunogenicity (4). Immunotherapy has significant advantages in improving the curative effect of melanoma, especially after the immune system is stimulated, and often result in a long-term curative effect and lasting immune memory (5). Therefore, tumor vaccine-induced immunotherapy has a favorable application prospect for the prevention and treatment of melanoma.

In eukaryotes, the ubiquitin-proteasome system (UPS) is critical to maintaining the intracellular protein balance and mediating specific irreversible protein degradation of short-lived intracellular proteins, as well as some non-functional proteins $(6,7)$. When certain proteins closely associated with apoptosis and cell cycle control are ubiquitin labelled, they can be identified and degraded by the $26 \mathrm{~S}$ proteasome (8-10). The effects of the UPS on cancer treatment have become a research hotspot. In 2004, scientists Aaron Ciechanover, Avram Hershko and Irwin Rose were awarded the Nobel Prize in Chemistry for the discovery of ubiquitin-mediated protein degradation (11). In 2009, articles on the antineoplastic effects of the UPS were published in Nature, where the prospects of such a system in cancer treatment were described from multiple points of view (12-17). Bortezomib, as the first drug targeting the UPS, passed clinical trials in 2003. After being approved by the Food and Drug Administration, it was selected as a therapeutic medicine for multiple malignant 
hematologic tumors, including relapsed or refractory multiple myeloma $(18,19)$. However, simply inhibiting the proteasome may cut off the entire UPS pathway. Consequently, not only is the transformation of a great number intracellular proteins inhibited, but significant adverse reactions are also observed (20). To reduce the influence on the other biological functions of the UPS, numerous regulatory enzymes that act on this pathway have become hot topics of research (20-22). At present, MLN4924, as a compound that targets an NEDD4 E3 ubiquitin protein ligase (NEDD4) activating enzyme, is at phase I of a clinical trial, and has shown promising antineoplastic activity $(23,24)$. Furthermore, melanoma treatment has been revolutionized by immuno- and targeted therapy (25-27).

NEDD4 is a type of ubiquitin-like protein, and its covalent bonding to a substrate is known as neddylation (28). An important physiological function of neddylation is to regulate E3 ubiquitin-ligating enzymes and promote the progression of the ubiquitination pathway $(29,30)$. Furthermore, the family of cullin proteins includes the substrates on which NEDD4 primarily acts. This family is one of the major core structures of E3 ubiquitin-ligating enzymes (31). As catalyzed by a series of enzymes, NEDD4 is covalently bound to cullin proteins, thereby forming a NEDD4 chain structure (31). Consequently, the confirmational changes to E3 ubiquitin-ligating enzymes, and E2 (which carries ubiquitin), promote entry to the E3-substrate complex, such that the substrate can be ubiquitinated (32). Numerous studies have reported that neddylation not only enables a significant increase in the ubiquitination efficiency of proteins to be degraded, but also promotes the degradation of these proteins by the $26 \mathrm{~S}$ proteasome $(33,34)$.

Previous studies have reported that NEDD4 serves an important role in cancer development $(29,30)$. NEDD4 is highly expressed in patients with breast (35) and lung cancer (36), where it was closely associated with clinical pathology $(35,36)$. Nevertheless, NEDD4 expression in melanoma, and its underlying mechanisms in melanoma occurrence and development, remain unknown. In the present study, NEDD4 expression in melanoma tissues and benign moles was detected via immunohistochemistry (IHC). Moreover, cellular experiments were conducted following NEDD4-knockout to determine its influence on the biological activity of melanoma cells, and to identify the associated underlying mechanisms.

\section{Materials and methods}

Patient samples, cell lines and cell culture. Specimens of 30 cases with cutaneous melanoma (CMM; stages I-II, 20 cases; and stages III-IV, 10 cases) and 10 cases with benign moles, surgically removed between March 2017 and March 2019, were selected from The Affiliated Xingtai People's Hospital of Hebei Medical University. The patients were not treated with radiotherapy, chemotherapy or immunotherapy prior to surgery. All patients were pathologically diagnosed with CMM after surgery, and the collected specimens were divided into two parts. Some specimens were frozen by diethyl pyrocarbonate (DEPC) water treatment, immersed in RNA Latter and stored at $-80^{\circ} \mathrm{C}$ for further use; the other specimens underwent paraffin embedding and slicing to
Table I. qPCR primer sequences.

\begin{tabular}{ll}
\hline Gene name & \multicolumn{1}{c}{ Primer sequence (5'-3') } \\
\hline $\begin{array}{l}\text { NEDD4 E3 } \\
\text { ubiquitin protein }\end{array}$ & F: TTGCACTTTGCAGCCAGAAG \\
ligase & \\
Notch1 & F: GCAGAGTGGGAGTCAGCTTCA \\
& R: GGTTGGTGAGGCAGGCATTGT \\
PTEN & F: GGTCTGAGTCGCCTGTCACCAT \\
& R:CCGTGTTGGAGGCAGTAGAAGG \\
GAPDH & F: CAAATTCCATGGCACCGTCA \\
& R: AGCATCGCCCCACTTGATTT
\end{tabular}

F, forward; R, reverse.

perform hematoxylin and eosin (H\&E) staining and IHC. The present study was approved by The Affiliated Xingtai People's Hospital Ethics Committee (approval no. 2017012205). All patients signed informed consent.

All cell lines used in the study were purchased from the American Type Culture Collection. Normal skin cells (HaCaT) and melanoma cell lines (SK-MEL-2, Malme-3M, MV3, A375 and MUM-2B cells) were all cultured in DMEM (Invitrogen; Thermo Fisher Scientific, Inc.) supplemented with $1 \%$ double-antibiotic $(100 \mathrm{U} / \mathrm{ml}$ penicillin and $100 \mathrm{mg} / \mathrm{l}$ streptomycin) and 10\% FBS (Gibco; Thermo Fisher Scientific, Inc.). The cells were maintained in an incubator at $37^{\circ} \mathrm{C}\left(5 \% \mathrm{CO}_{2}\right)$. After 3 days, the cell confluence had reached $80-90 \%$, and the cells were passaged.

Reagents and instruments. A reverse transcription (RT) kit and PCR kit were purchased from Takara Biotechnology Co., Ltd., while DEPC was from Sangon Biotech Co., Ltd. TRIzol ${ }^{\circledR}$ reagent was purchased from Invitrogen (Thermo Fisher Scientific, Inc.). Richard-Allan Scientific ${ }^{\mathrm{TM}}$ Wright-Giemsa compound stain was acquired from Thermo Fisher Scientific, Inc., and the Annexin V-FITC/PI apoptosis assay kit was from Nanjing KeyGen Biotech Co., Ltd. The Transwell assay was obtained from Corning, Inc., and crystal violet from Sigma-Aldrich; Merck KGaA. NEDD4 (cat. no. ab46521), PTEN (cat. no. ab170941), Notch1 (cat. no. ab52627) and GAPDH (cat. no. ab181602) antibodies were acquired from Abcam. FBS were all purchased from Gibco; Thermo Fisher Scientific, Inc. Small interfering RNA (siRNA/si)-NEDD4 was purchased from Nanjing KeyGen Biotech Co., Ltd. Lipofectamine $^{\circledR} 3000$ (Invitrogen; Thermo Fisher Scientific, Inc.) was used for transfection, and all PCR primers were purchased from Sangon Biotech Co., Ltd.

$H \& E$ staining. Deparaffination and rehydration were performed using the following conventional methods. The sections $(5-\mu \mathrm{m})$ were immersed in xylene for $5 \mathrm{~min}$ at room temperature, which was then replaced for immersion for another $5 \mathrm{~min}$. The sections were then rehydrated with a descending alcohol series (absolute, 95\%, 85\% and 70\% ethyl alcohol for 5 min each), following by washing in PBS thrice for 3 min each. Next, the sections were immersed in nuclear staining solution (Reagent I 
from the H\&E kit; cat. no. KGA224; Nanjing KeyGen Biotech Co., Ltd.) for 3-5 min at room temperature, and then in color separation fluid I of Reagent II for $20 \mathrm{sec}$, followed by washing for 30-60 sec. Subsequently, the sections were immersed in color separation fluid II of Reagent III for $\sim 40 \mathrm{sec}$, and then washed with water for 30-60 sec at room temperature, prior to staining for $2 \mathrm{~min}$ in Reagent IV. Reagent $\mathrm{V}$ was used to wash the sections twice, and excess solution was removed. After the sections were dried via absorption using filter paper, they were mounted, underwent light-microscopic examination and were imaged at x400 magnification.

IHC staining. Tissue sections (thickness, 3- $\mu \mathrm{m}$ ) underwent deparaffination $\left(60^{\circ} \mathrm{C} ; 2 \mathrm{~h}\right)$, dehydration using a gradient ethanol series (absolute ethanol, 95\% ethanol, 85\% ethanol and $70 \%$ ethanol all for $5 \mathrm{~min}$ ) and antigen retrieval at room temperature, which involved thermally induced epitope repair in citrate buffer ( $\mathrm{pH} \mathrm{6.0)}$ for $30 \mathrm{~min}$. The sections were sealed with $\mathrm{H}_{2} \mathrm{O}_{2}$ at room temperature for $2 \mathrm{~h}$, and then incubated with primary (cat. no. ab46521; Abcam) and secondary (cat. no. KGAA35; Nanjing KeyGen Biotech Co., Ltd.) antibodies at $4^{\circ} \mathrm{C}$ for $12 \mathrm{~h}$, and at $37^{\circ} \mathrm{C}$ for $2 \mathrm{~h}$, respectively. Next, DAB staining was conducted for $5 \mathrm{~min}$ at room temperature and nuclear staining (hematoxylin) at room temperature for $5 \mathrm{~min}$, followed by mounting for subsequent light-microscopic examination (magnification, $\mathrm{x} 400$ ); five visual fields were randomly select to determine the corresponding relative optical density. The data were analyzed using Quantity One software v.4.66 (Bio-Rad Laboratories, Inc.).

Reverse transcription-quantitative $(R T-q) P C R$. Total RNA was extracted from clinical specimens and cells using TRIzol ${ }^{\circledR}$ reagent (Invitrogen; Thermo Fisher Scientific, Inc.) which were centrifuged for $5 \mathrm{~min}$ at $12,000 \mathrm{x} \mathrm{g}$ at $4^{\circ} \mathrm{C}$. The corresponding supernatant was harvested. Next, $1 \mathrm{ml} \mathrm{75 \%} \mathrm{ethyl} \mathrm{alcohol} \mathrm{was}$ added to $1 \mathrm{ml} \mathrm{TRIzol}{ }^{\circledR}$ (Invitrogen; Thermo Fisher Scientific, Inc.), and a NanoDrop 2000 spectrophotometer (NanoDrop Technologies; Thermo Fisher Scientific, Inc.) was used to calculate the RNA concentration after 5-10 min of drying at room temperature (or vacuum drying). The real-time PCR Master Mix (SYBR-Green; Takara Bio, Inc.) and the ABI StepOne plus real-time PCR system (Applied Biosystems; Thermo Fisher Scientific, Inc) were used for qPCR. The following thermocycling conditions were used: Initial denaturation at $95^{\circ} \mathrm{C}$ for $10 \mathrm{~min}$; followed by 40 cycles of denaturation at $95^{\circ} \mathrm{C}$ for $15 \mathrm{sec}$ and annealing at $60^{\circ} \mathrm{C}$ for $1 \mathrm{~min}$; and a final extension of $10 \mathrm{~min}$ at $72^{\circ} \mathrm{C}$ and amplification was monitored in a real-time manner. GAPDH was used as the reference gene. For each sample, the test was repeated three times, and sample gene expression was quantified using the $2^{-\Delta \Delta C q}$ (37) method. The primer sequences are presented in Table I.

Transfection. A day before transfection, cells were seeded into a culture plate. Culture medium without antibiotics was added into each well, such that the confluence had reached $70-80 \%$ at the time of transfection. Then, $2.5 \mu \mathrm{g}$ siRNA was diluted using $250 \mu \mathrm{l}$ Opti-MEM (Thermo Fisher Scientific, Inc) without serum, followed by gentle mixing and incubation at room temperature for $5 \mathrm{~min}$. Then, $5 \mu \mathrm{l}$ Lipofectamine ${ }^{\circledR} 3000$ was diluted in $250 \mu \mathrm{l}$ Opti-MEM (without serum), mixed and incubated at room temperature for $5 \mathrm{~min}$. Next, the siRNA-Lipofectamine mixture was added to each well (containing $500 \mu \mathrm{l}$ culture media and cells), and cultured for 4-6 h in DMEM at $37^{\circ} \mathrm{C}$. The culture medium was replaced with a fresh medium (10\% FBS), and the cells were placed in a $5 \% \mathrm{CO}_{2}$ incubator $\left(37^{\circ} \mathrm{C}\right)$ for $48 \mathrm{~h}$. The siRNA sequences were as follows: si-NEDD4-1 forward, 5'-GGGAAGAGA GGCAGGAUAUTT-3' and reverse, 5'-AUAUCCUGCCUC UCUUCCCTT-3'; si-NEDD4-2 forward, 5'-CCUAACAGAU GCUGAGAAUTT-3' and reverse, 5'-AUUCUCAGCAUC UGUUAGGTT-3'; si-NEDD4-3 forward, 5'-GUGAAAAGG GAUUGGAUUATT-3' and reverse, 5'-UAAUCCAAUCCC UUUUCACTT-3'; and si-negative control (NC) forward, 5'-UGACCUACAACUUCUAUGGTT-3' and reverse, 5'-UUC UCCGAACGUGUCACGUTT-3'. Subsequent experimentation was performed $48 \mathrm{~h}$ after transfection.

Plate and soft agar colony formation experiments. Cells in the logarithmic phase were harvested and counted, and the concentration was adjusted to $1 \times 10^{3} / \mathrm{ml}$. In total, $3 \times 10^{3}$ cells were collected and placed in culture medium. Distilled water was used to prepare 1.3 and $0.8 \%$ agarose solutions with low melting points. After the agarose solution was mixed with RPMI-1640 (MilliporeSigma) at a 1:1 ratio, the mixture was evenly distributed on a plate where it was naturally cooled and solidified into a double agar layer. Next, $1 \mathrm{ml}$ cell suspension was added to the solidified upper agar layer, and the plate was placed in an incubator $\left(37^{\circ} \mathrm{C}\right)$ for $\sim 2$ weeks. Both colony morphology observation and counting were performed. For both the experimental and control groups, the test was repeated thrice.

Flow cytometry. Once the supernatant of the transfected cells was removed, double staining was performed using the Annexin V-FITC/PI apoptosis assay kit (BD Biosciences) per the manufacturer's protocol. Apoptosis was analyzed via flow cytometry. Moreover, the cells were divided into live cells, non-viable non-apoptotic cells, viable apoptotic cells and non-viable apoptotic cells. The number of apoptotic cells was determined using the Muse Cell Analyzer (EMD Millipore). The Muse software v.1.1.2 (EMD Millipore) evaluated the numbers of apoptotic rate.

Transwell assay. The transfected cells $\left(1 \times 10^{4}\right.$ cells/well) were seeded into the upper chamber of a Transwell insert pre-coated with Matrige ${ }^{\circledR}$ (Corning, Inc.) in serum-free medium. DMEM containing $15 \%$ FBS was added into the 96-well plate, and $100 \mu \mathrm{l}\left(1 \times 10^{6} / \mathrm{ml}\right)$ cell suspension was added to the upper chambers. After continuous cultivation for $24 \mathrm{~h}$ in an incubator, the inserts were removed and washed with PBS, and the redundant cells were removed using cotton swabs. Then, the membranes were fixed for 15 min with $4 \%$ paraformaldehyde at room temperature, dried and stained for $15 \mathrm{~min}$ with $0.5 \%$ crystal violet at $37^{\circ} \mathrm{C}$. After washing three times with PBS, the plate was assessed under a light microscope, images were captured, and the number of invasive cells was calculated.

Wound-healing assay. The cells were inoculated into a 6-well plate $\left(2 \times 10^{5} / \mathrm{ml} ; 2 \mathrm{ml} /\right.$ well $)$. When reaching $80-90 \%$ confluency, the supernatant was removed, which was followed by washing 
A
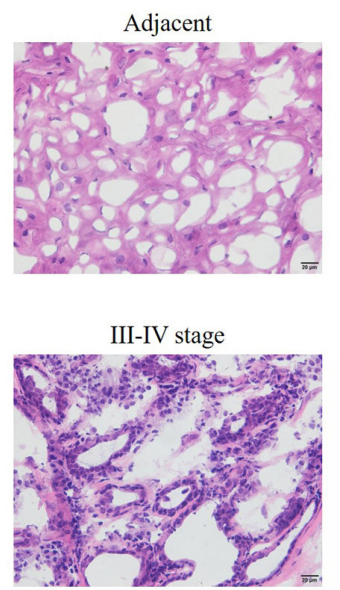

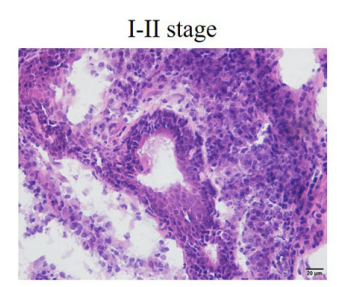

B
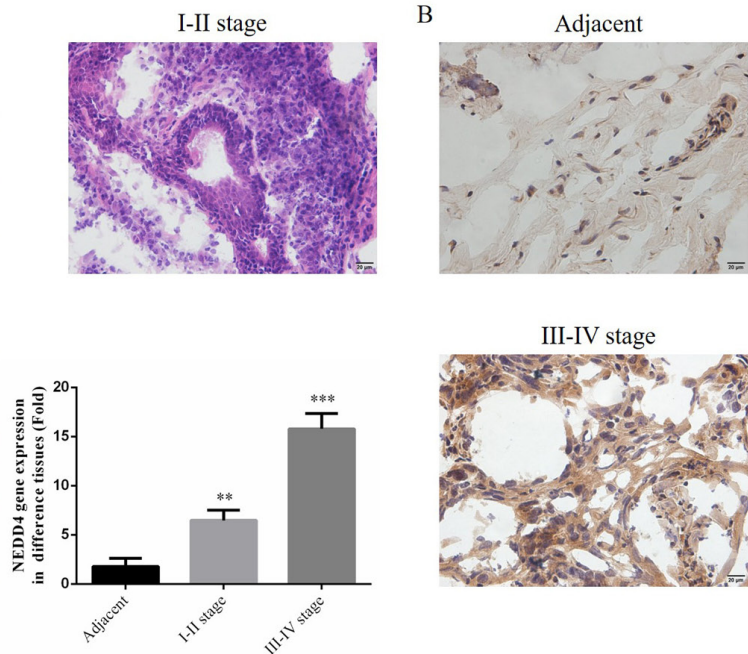

III-IV stage
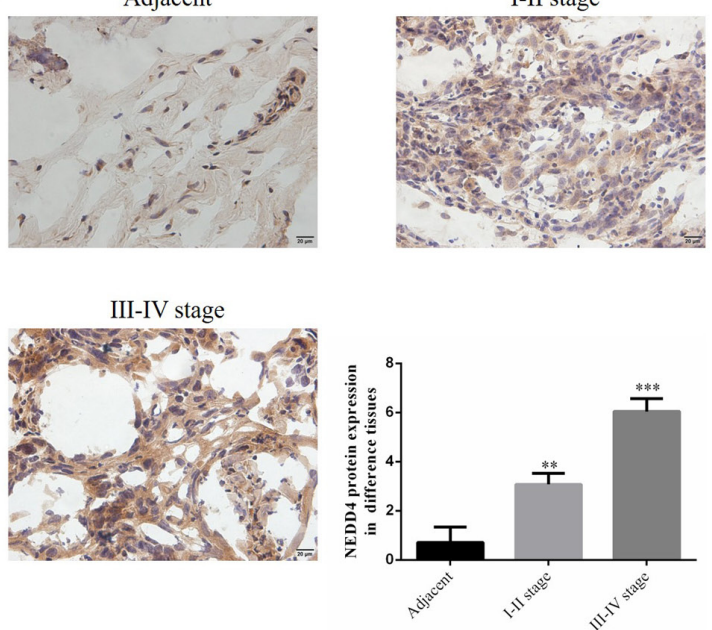

Figure 1. Pathological changes and NEDD4 mRNA and protein expression in cutaneous melanoma clinical samples. (A) Pathology of different tissues was detected by hematoxylin and eosin staining; magnification, x400. NEDD4 mRNA expression was measured using reverse transcription-quantitative PCR. (B) NEDD4 protein expression in difference tissues was examined using immunohistochemistry; magnification, $\mathrm{x} 400$. ${ }^{* *} \mathrm{P}<0.01$ and ${ }^{* * * *} \mathrm{P}<0.001$ vs. adjacent normal tissues. Adjacent, adjacent normal tissues; I-II stage, I-II stage melanoma; III-IV stage, III-IV stage melanoma. NEDD4, NEDD4 E3 ubiquitin protein ligase.

once with PBS. Then, the monolayers were perpendicularly scratched along the median line using a $10-\mu 1$ pipette tip. After washing twice with PBS, serum-free culture medium was added to the wells, and images were captured at 0,24 and $48 \mathrm{~h}$ under a light microscope (x100). The corresponding wound-closure distance was calculated as wound healing rate, using the following calculation: $(0 \mathrm{~h}$ width $-24 \mathrm{~h}$ or $48 \mathrm{~h}$ width)/0 h width x $100 \%$.

Western blotting. After the proteins were extracted from different groups of cells, which were treated with different molecules using RIPA lysis buffer (Beyotime Institute of Biotechnology), their concentration was determined using a BCA assay. 10\% SDS-PAGE was performed to separate the proteins (50 $\mu \mathrm{g}$ per lane), which were then transferred onto a PVDF membrane. After washing with TBS-Tween-20 (TBST; $0.1 \%$ ), skimmed milk was used to block the membrane for $2 \mathrm{~h}$ at room temperature. Then, primary antibodies against NEDD4, PTEN, Notch1 and GAPDH (Abcam) were added to the membrane, which was incubated at $4^{\circ} \mathrm{C}$ overnight. Then, the membrane was rewarmed for $\sim 30 \mathrm{~min}$ at room temperature, washed with TBST, and incubated with the horseradish peroxidase conjugated goat anti rabbit secondary antibody (1:5,000; cat. no. abs20002; Absin Bioscience, Inc.) at $37^{\circ} \mathrm{C}$ for $2 \mathrm{~h}$ on a shaker at room temperature with secondary antibody. DAB was used for color development, which was exposed using an imager. The bands were detected using an ECL reagent (EMD Millipore). The grayscale values of the membranes were semi quantified using ImageJ software v.1.52r (National Institutes of Health) and the relative expression differences were determined using GAPDH as the reference. The antibody dilutions were as follows: NEDD4, 1:100; Notch1, 1:100; PTEN, 1:200; and GAPDH, 1:500.

Statistical analysis. Statistical analysis was performed using SPSS 22.0 software (IBM Corp.), and the data are expressed as the mean \pm SD. Statistical analysis was conducted using one-way ANOVA and RMANOVA followed by Tukey's post hoc test. All experiments were repeated 3 times, and $\mathrm{P}<0.05$ was considered to indicate a statistically significant difference.

\section{Results}

Pathological changes and NEDD4 $m R N A$ and protein expression in CMM clinical samples. Using H\&E staining, it was identified that immune cell infiltration were increased in cancer tissues as the stage increased, and there was no infiltration of the benign nevus. With clear interstitial boundaries, NEDD4 mRNA expression was significantly upregulated compared with that of the adjacent normal tissues, as measured by RT-qPCR ( $<<0.01$; Fig. 1A). Using IHC, it was found that, compared with adjacent tissues, NEDD4 protein expression was significantly enhanced in tumor tissues $(\mathrm{P}<0.01$; Fig. 1B), and NEDD4 protein expression at stages III-IV tissues was higher than that at stages I-II. The aforementioned results indicated that NEDD4 was associated with melanoma. NEDD4 might be associated with CMM.

NEDD4 gene expression in different cell lines and siRNA treatment groups. Compared with normal epidermal HaCaT cells, NEDD4 gene expression was significantly upregulated in melanoma cell lines (SK-MEL-2, Malme-3M, MV3, A375 and MUM-2B) $(\mathrm{P}<0.01)$, and was highest in SK-MEL-2 and Malme-3M cells (Fig. 2A). accordingly, SK-MEL-2 and Malme-3M cells were selected for subsequent experimentation. In total, three types of siRNA that had knockdown effects on NEDD4 expression were used. NEDD4 mRNA expression was significantly suppressed in the siRNA-transfected SK-MEL-2 and Malme-3M cells compared with the si-NC groups $(\mathrm{P}<0.01$; Fig. 2B). Moreover, NEDD4 mRNA expression in the siRNA-2 group was the lowest in both the SK-MEL-2 and Malme-3M cell lines. After siRNA-2 transfection (compared with the NC group), NEDD4 mRNA expression was significantly decreased in SK-MEL-2 and Malme-3M cell lines $(\mathrm{P}<0.001$, 
A

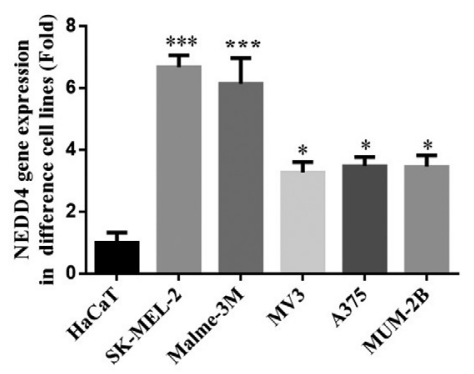

$\mathrm{B}$
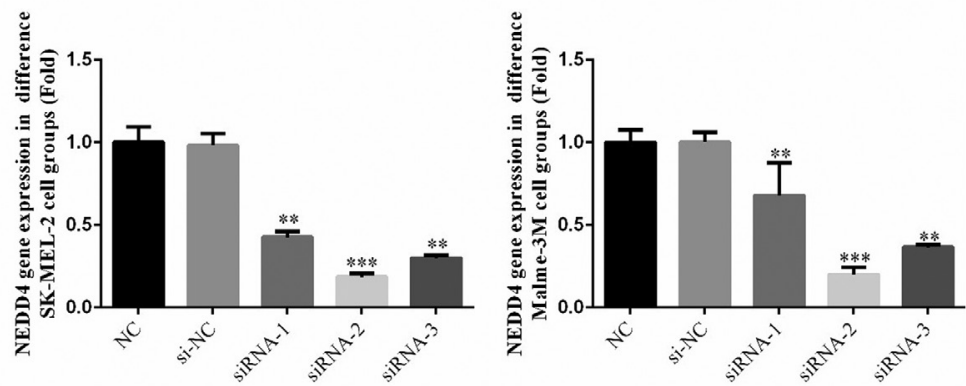

Figure 2. NEDD4 gene expression in different melanoma cell lines and transfection groups. (A) NEDD4 gene expression in different cell lines was detected by RT-qPCR. "P $<0.05$ and ${ }^{* * * *} \mathrm{P}<0.001$ vs. the HaCaT cell line. (B) NEDD4 gene expression in different transfection groups in SK-MEL-2 and Malme-3M cells was measured by RT-qPCR. ${ }^{* *} \mathrm{P}<0.01$ and ${ }^{* * *} \mathrm{P}<0.001$ vs. the NC group. NC, cells treated with culture medium; si-NC, cells transfected with si-NC; siRNA-1-3 cells transfected with si-NEDD4-1-3; NC, negative control; siRNA/si, small interfering RNA; NEDD4, NEDD4 E3 ubiquitin protein ligase; RT-qPCR, reverse transcription-quantitative PCR.

A

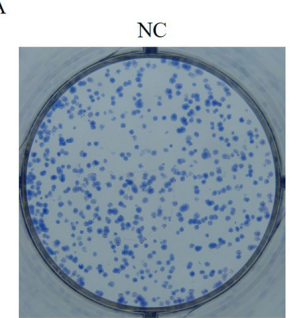

si-NEDD4

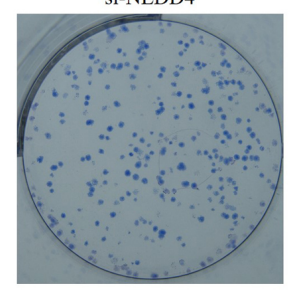

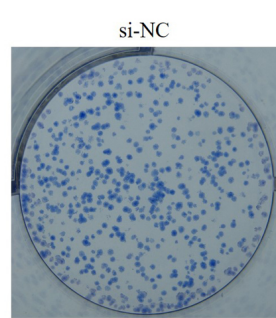

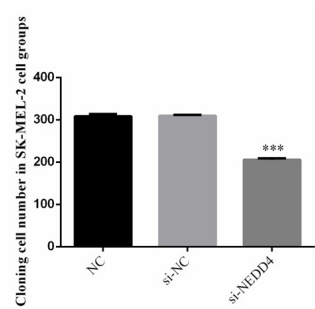

B

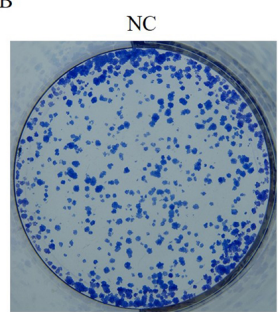

si-NEDD4

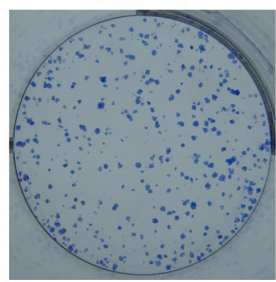

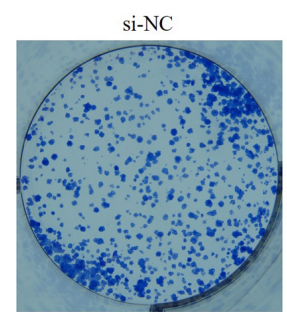

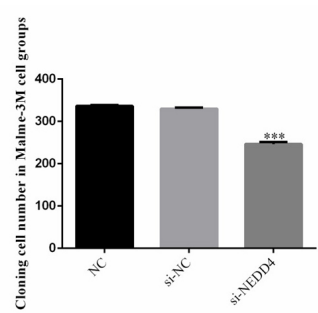

Figure 3. NEDD4-knockdown suppresses cellular proliferation. Colony number in the (A) SK-MEL-2 and (B) Malme-3M cell groups. ${ }^{* * *} \mathrm{P}<0.001$ vs. the NC group. NC, cells treated with culture medium; si-NC, cells transfected with si-NC; si-NEDD4, cells transfected with si-NEDD4. NC, negative control; siRNA/si, small interfering RNA; NEDD4, NEDD4 E3 ubiquitin protein ligase.

Fig. S1). From the results, the SK-MEL-2 and Malme-3M cell lines were selected for the further experiments, and si-RNA-2 could decrease the expression level of NEDD4 in these cell lines.

NEDD4-knockdown suppresses cellular proliferation. Following si-NEDD4 transfection, the colony cell number was significantly decreased compared with that of the NC group $(\mathrm{P}<0.001$; Fig. 3A and B) in SK-MEL-2 and Malme-3M cell lines. NEDD4 knockdown could suppress cell proliferation in the SK-MEL-2 and Malme-3M cell lines.

NEDD4-knockdown increases apoptosis. Using flow cytometry, it was found that the cell apoptotic rate of the si-NEDD4 groups was significantly increased $(\mathrm{P}<0.001$; Fig. 4A and $\mathrm{B})$ compared with that of the NC groups in SK-MEL-2 and Malme-3M cells. NEDD4 knockdown could increase cell apoptosis in the SK-MEL-2 and Malme-3M cell lines. 
A
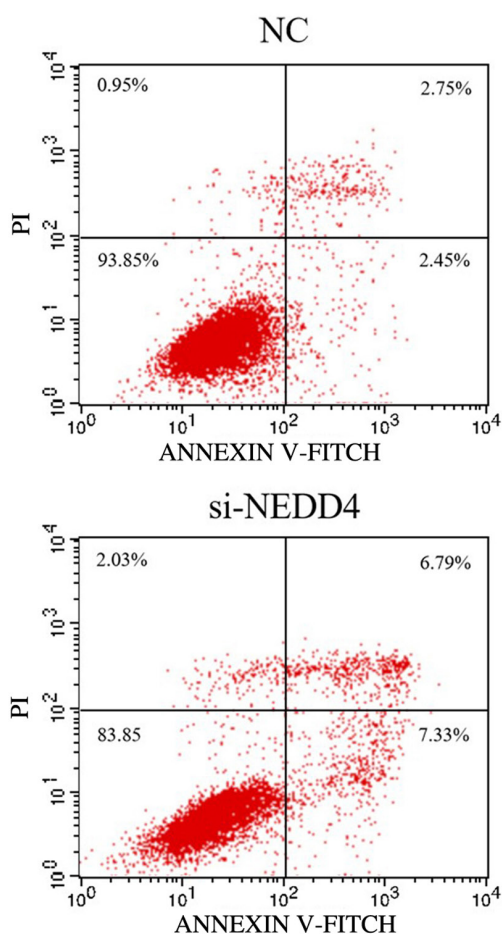

B

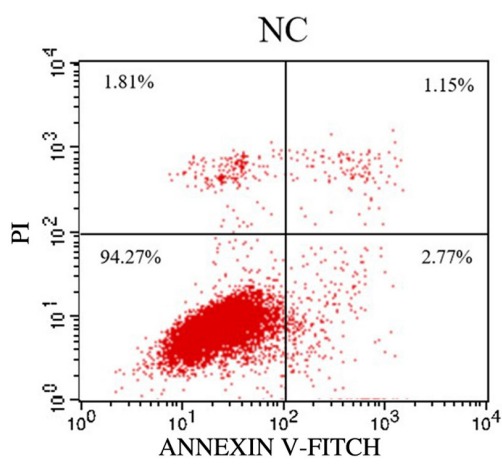

si-NEDD4

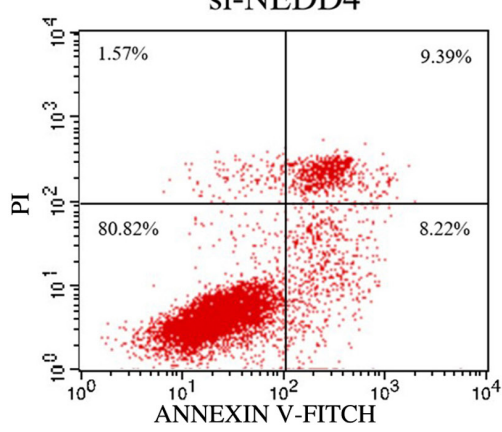

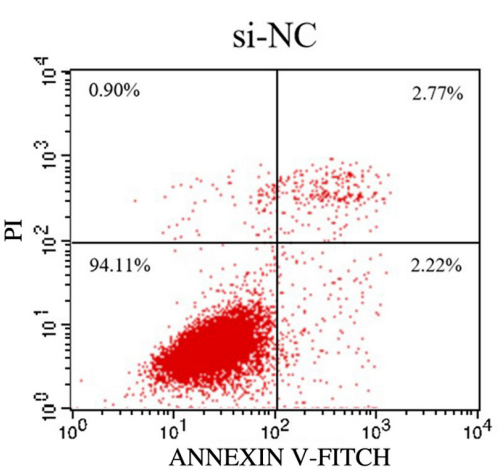
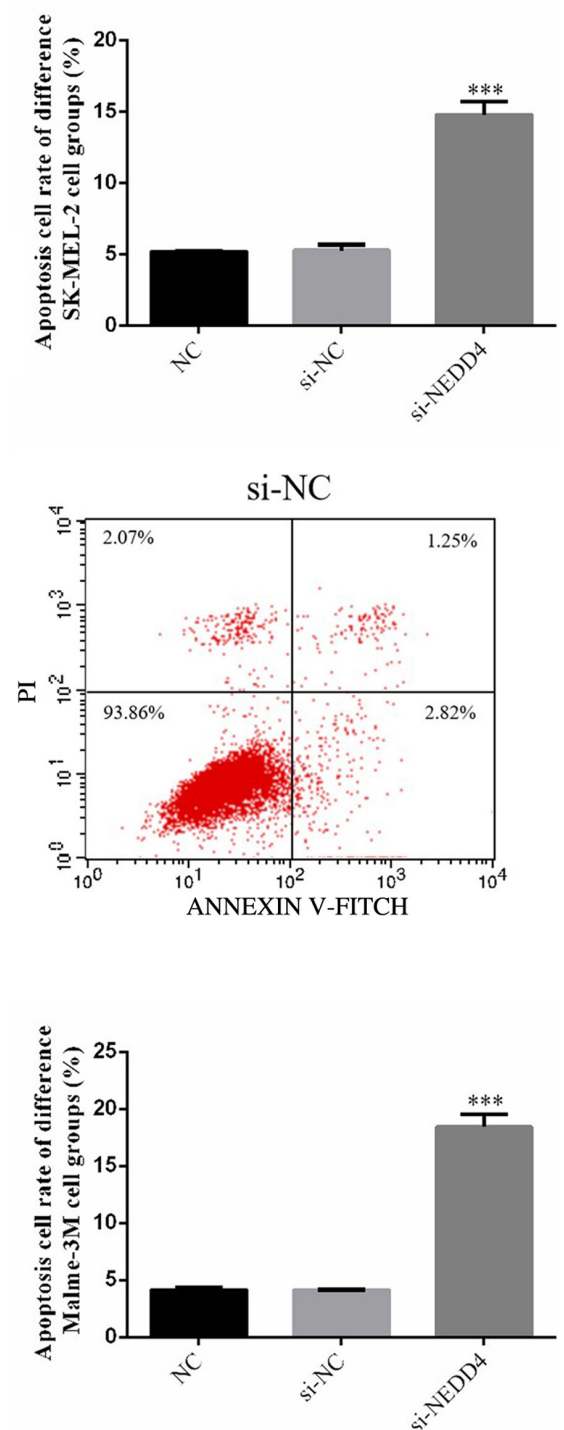

Figure 4. NEDD4-knockdown increases cell apoptotic rate. NEDD4-knockdown increased the apoptosis of (A) SK-MEL-2 and (B) Malme-3M cells, as determined by flow cytometry. ${ }^{* * *} \mathrm{P}<0.001$ vs. the NC group. NC, cells treated with culture medium; si-NC, cells transfected with si-NC; si-NEDD4, cells transfected with si-NEDD4. NC, negative control; siRNA/si, small interfering RNA; NEDD4, NEDD4 E3 ubiquitin protein ligase.

NEDD4-knockdown inhibits cellular invasiveness. Using a Transwell assay, it was demonstrated that the invasive cell number in the si-NEDD4 groups was significantly decreased $(\mathrm{P}<0.001$; Fig. 5A and $\mathrm{B})$ compared with that in the $\mathrm{NC}$ groups in both the SK-MEL-2 and Malme-3M cell lines. Following NEDD4 knockdown, the invasion abilities of the SK-MEL-2 and Malme-3M cell lines were significantly decreased

NEDD4-knockdown decreases wound-healing capacity. The wound-healing assay results revealed that the closure rate of the si-NEDD4 groups, in which NEDD4 was knocked down 

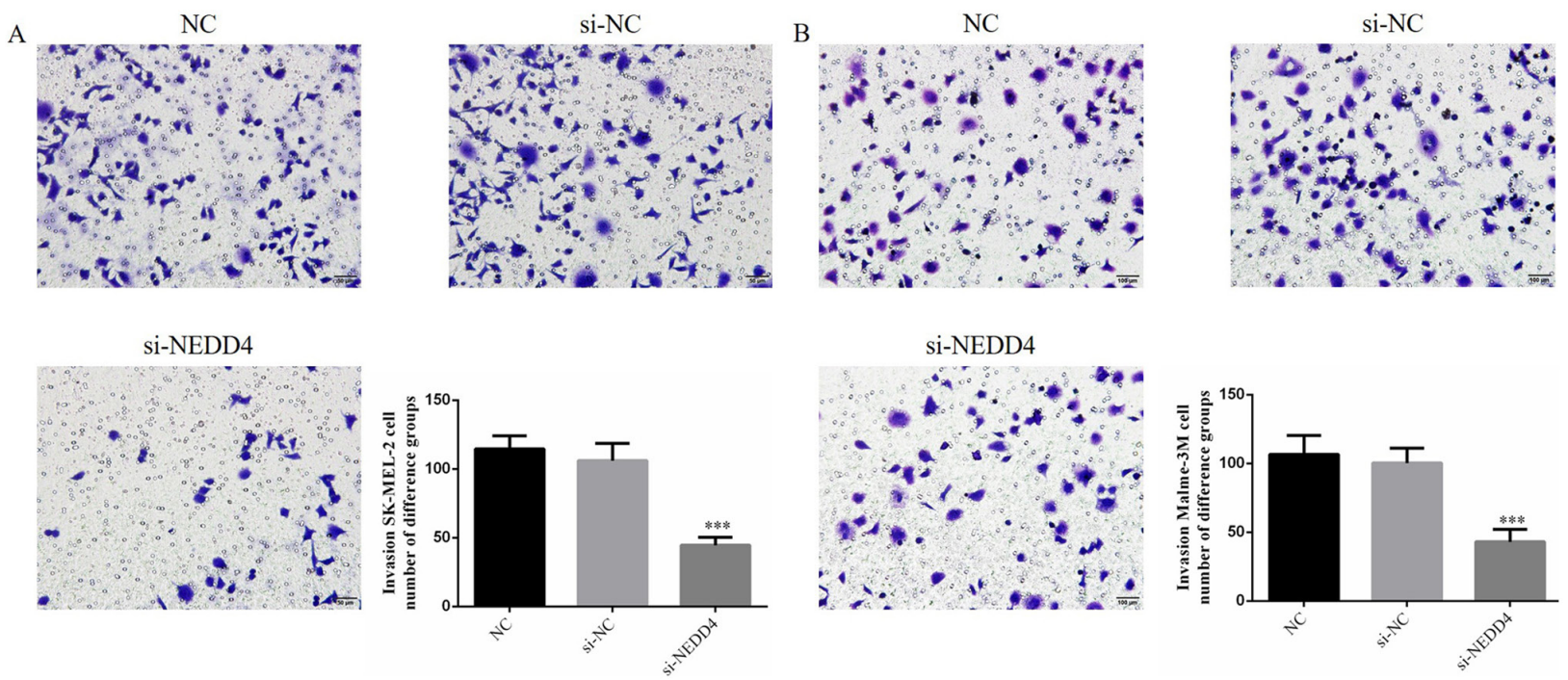

Figure 5. NEDD4-knockdown decreases cellular invasive capacity. (A) SK-MEL-2 and (B) Malme-3M cell numbers in different groups, as determined using a Transwell assay. ${ }^{* * *} \mathrm{P}<0.001$ vs. the NC group. NC, cells treated with culture medium; si-NC, cells transfected with si-NC; si-NEDD4, cells transfected with si-NEDD4. NC, negative control; siRNA/si, small interfering RNA; NEDD4, NEDD4 E3 ubiquitin protein ligase.
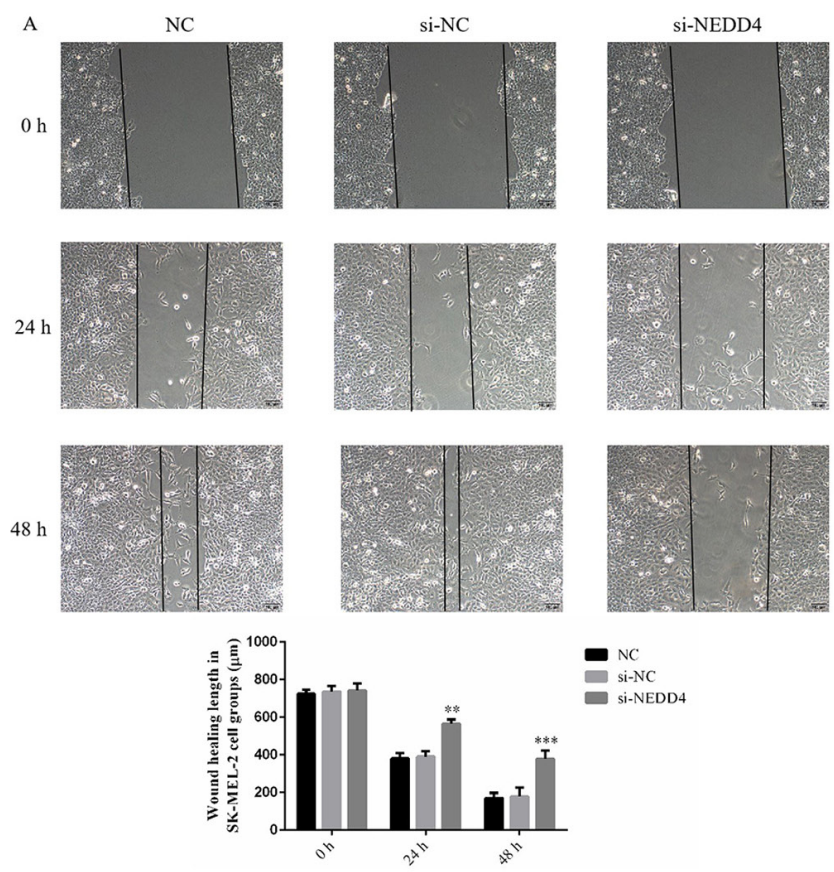
si-NC
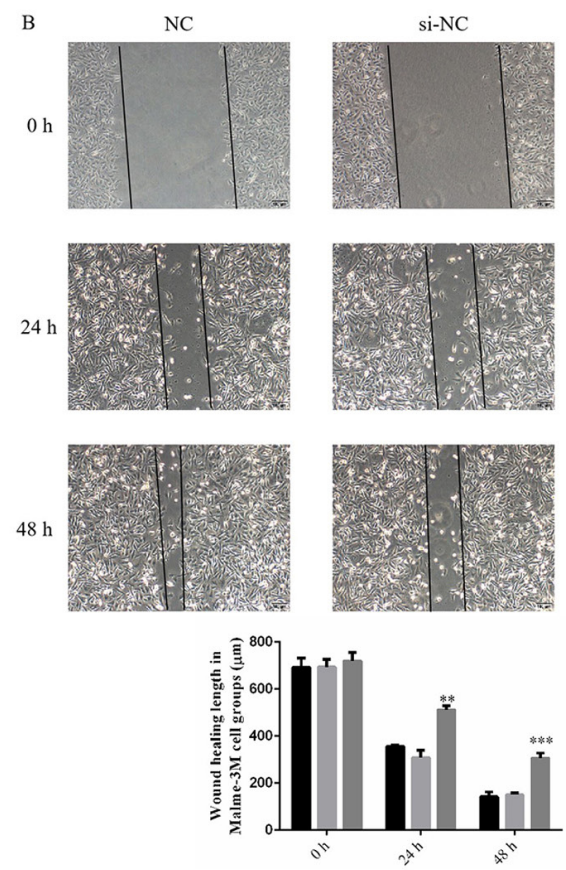
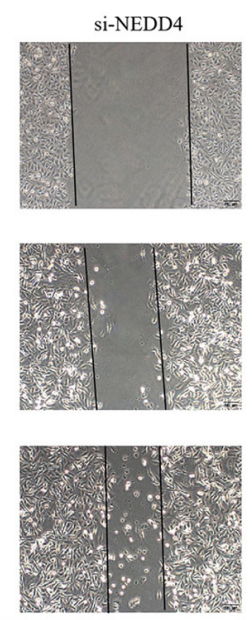

-

Figure 6. NEDD4-knockdown affects wound-healing in different groups. Wound width in (A) SK-MEL-2 and (B) Malme-3M cell groups. ${ }^{* *}<<0.01$ and ${ }^{* * *} \mathrm{P}<0.001$ vs. the NC group. NC, cells treated with culture medium; si-NC, cells transfected with si-NC; si-NEDD4, cells transfected with si-NEDD4. NC, negative control; siRNA/si, small interfering RNA; NEDD4, NEDD4 E3 ubiquitin protein ligase.

by si-NEDD4 transfection, was significantly suppressed at 24 and $48 \mathrm{~h}(\mathrm{P}<0.01$; Fig. $6 \mathrm{~A}$ and $\mathrm{B})$ compared with that in the NC group in both the SK-MEL-2 and Malme-3M cell lines. Following NEDD4 knockdown, the cell migration abilities of the SK-MEL-2 and Malme-3M cell lines were significantly decreased.

NEDD4-knockdown affects the relative gene expression of Notch 1 and PTEN. From RT-qPCR analysis, the results demonstrated that NEDD4 and Notch1 gene expression was significantly decreased $(\mathrm{P}<0.001$; Fig. 7A and $\mathrm{B})$, while
PTEN gene expression was significantly increased in the si-NEDD4 groups $(\mathrm{P}<0.001$; Fig. 7A and $\mathrm{B})$ compared with those of the NC SK-MEL-2 and Malme-3M cell groups. NEDD4 knockdown might be regulated to Notch1 and PTEN gene expression in the SK-MEL-2 and Malme-3M cell lines.

NEDD4-knockdown affects the relative protein expression of Notch 1 and PTEN. The western blotting results indicated that NEDD4 and Notch-1 protein expression was significantly downregulated ( $\mathrm{P}<0.001$; Fig. 8$)$, and $\mathrm{PTEN}$ protein expression 
A
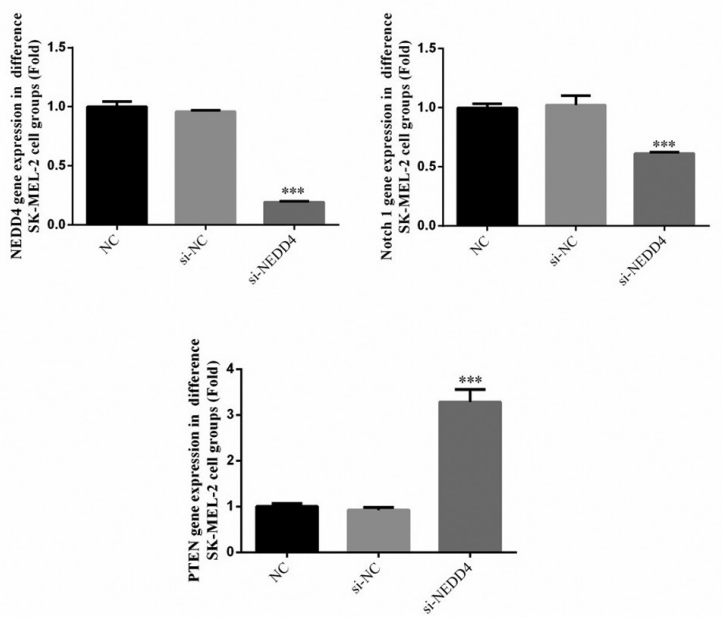
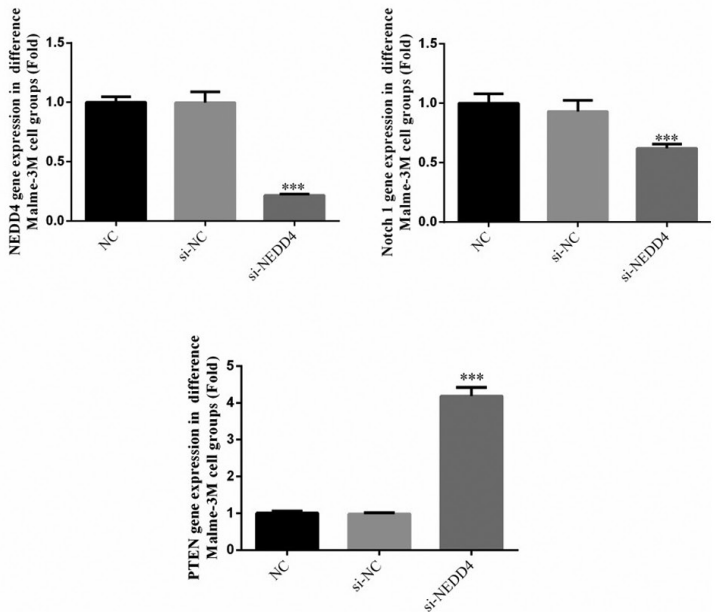

Figure 7. NEDD4-knockdown the affects relative gene expression of Notch 1 and PTEN. Relative mRNA expression of Notch 1 and PTEN in (A) SK-MEL-2 and (B) Malme-3M cell groups. ${ }^{* * *} \mathrm{P}<0.001$ vs. the NC group. NC, cells treated with culture medium; si-NC, cells transfected with si-NC; si-NEDD4, cells transfected with si-NEDD4. NC, negative control; siRNA/si, small interfering RNA; NEDD4, NEDD4 E3 ubiquitin protein ligase; Notch 1, notch receptor 1.

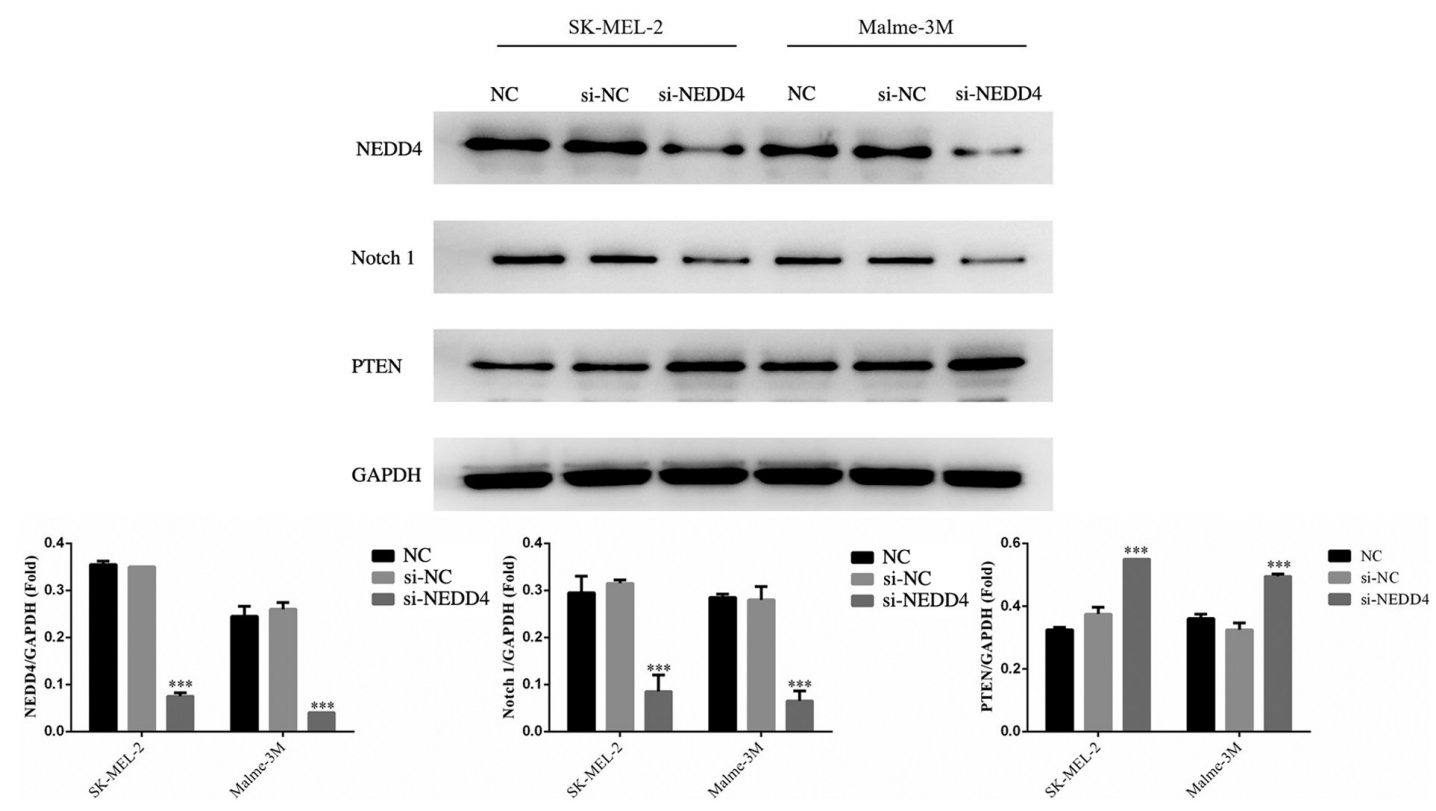

Figure 8. NEDD4-knockdown affects the relative protein expression of Notch 1 and PTEN. Notch 1 and PTEN expression levels were determined by western blotting. ${ }^{* * *} \mathrm{P}<0.001$ vs. the NC group. NC, cells treated with culture medium; si-NC, cells transfected with si-NC; si-NEDD4, cells transfected with si-NEDD4. NC, negative control; siRNA/si, small interfering RNA; NEDD4, NEDD4 E3 ubiquitin protein ligase; Notch 1, notch receptor 1.

was significantly increased in the si-NEDD4 groups $(\mathrm{P}<0.001$; Fig. 8), compared with those of the NC group, in both SK-MEL-2 and Malme-3M cells. NEDD4 knockdown might be regulated to Notch1 and PTEN protein expression in the SK-MEL-2 and Malme-3M cell lines.

\section{Discussion}

Over the past few years, studies have reported that NEDD4 serves an essential role in tumor development (33-36). For example, relevant research findings have shown that abnormal expression of NEDD4 is closely associated with the bioactivity and prognosis of tumors (38-41). However, to date, neither the expression nor relevant underlying mechanisms of NEDD4 in melanoma have been extensively investigated. In the present study, IHC and RT-qPCR analyses were initially used to quantify NEDD4 expression in normal pigment nevus and CMM tissues. After NEDD4-knockdown, not only was the bioactivity of melanoma observed, but the corresponding mechanism was investigated based on molecular biological experiments.

The present results demonstrated that the gene and protein expression levels of NEDD4 in CMM tissues were significantly increased compared with the normal pigment nevus tissues. Moreover, as the stage increased,NEDD4 expression consistently increased. In the cellular experiments, the bioactivity (e.g., 
proliferation, invasion and migration ability) of CMM cells (SK-MEL-2 and Malme-3M; with highly expressed NEDD4) was decreased following NEDD4-knockdown. As shown by western blotting and RT-qPCR, the mRNA and protein expression levels of NEDD4 and Notch1 were significantly downregulated, while those of PTEN were significantly increased.

At present, the Notch signal transduction pathway is considered a research hotspot in the life sciences field. As one of the major signaling pathways that mediates cell-to-cell contact, it is capable of regulating cellular proliferation, differentiation and apoptosis $(42,43)$. Moreover, NEDD4-silencing was reported to effectively suppress Notch1 expression (44). Additionally, Notch1 has a reverse regulatory effect on downstream PTEN genes $(42,45)$, and PTEN deleted on chromosome 10 was the first tumor suppressor gene found to possess a dual phosphatase activity. PTEN mutation or expression loss is closely associated with the occurrence and development of multiple malignant tumors (46). Furthermore, PTEN expression loss is an important promoter of tumor occurrence. In the present study, it was found that PTEN expression was increased, along with a reduction in Notch1 expression, following NEDD4-knockdown. However, there were some study limitations. Using siRNA to knockdown NEDD4 expression in SK-MEL-2 and Malme-3M cells, proliferation significantly decreased, while apoptosis significantly increased. However, invasion and migration ability were significantly downregulated. Thus, the effects of apoptosis on invasion and migration cannot be ruled out. Furthermore, the study only discussed the effects of the NEDD4/Notch1/PTEN pathway in melanoma development, and other pathways may also be implicated, which will be investigated in future research.

In conclusion, the present study demonstrated that interference with NEDD4 expression has the potential to effectively inhibit the proliferative abilities of CMM cells, as well as inhibit invasive and migratory capacity. Moreover, the underlying mechanism of NEDD4 may be closely associated with the regulation of the downstream Notch1/PTEN signaling pathway.

\section{Acknowledgements}

Not applicable.

\section{Funding}

No funding was received.

\section{Availability of data and materials}

The datasets used and/or analyzed during the current study are available from the corresponding author on reasonable request.

\section{Authors' contributions}

All authors contributed to the study conception and design. Material preparation, data collection and analysis were performed by FC, YC, XZ, LA, LY, ZL and LZ. The first draft of the manuscript was written by FC and all authors commented on previous versions of the manuscript. FC and
RH confirm the authenticity of all the raw data. All authors read and approved the final manuscript.

\section{Ethics approval and consent to participate}

The study was approved by The Affiliated Xingtai People's Hospital Ethics Committee (Xingtai, China) and informed consent was signed by all participants (approval no. 2017012205).

\section{Patient consent for publication}

Not applicable.

\section{Competing interests}

The authors declare that they have no competing interests.

\section{References}

1. Riley RS, June CH, Langer R and Mitchell MJ: Delivery technologies for cancer immunotherapy. Nat Rev Drug Discov 18: 175-196, 2019.

2. National Cancer Institute (NCI): SEER Stat Fact Sheet: Melanoma of the skin. http://seer.cancer.gov/statfacts/html/melan.html. Accessed June 20, 2015

3. Joshua AM, Evans A, Van der Kwast T, Zielenska M, Meeker AK, Chinnaiyan A and Squire JA: Prostatic preneoplasia and beyond. Biochim Biophys Acta 1785: 156-181, 2008.

4. Hershko A: The ubiquitin system for protein degradation and some of its roles in the control of the cell-division cycle (Nobel lecture). Angew Chem Int Ed 44: 5932-5943, 2005.

5. Lee AY and Brady MS: Neoadjuvant immunotherapy for melanoma. J Surg Oncol 123: 782-788, 2021.

6. Hjerpe R and Rodríguez MS: Alternative UPS drug targets upstream the $26 \mathrm{~S}$ proteasome. Int J Biochem Cell Biol 40: 1126-1140, 2008.

7. Tsvetkov P, Reuven N and Shaul Y: Ubiquitin-independent p53 proteasomal degradation. Cell Death Differ 17: 103-108, 2010.

8. Allende-Vega N and Saville MK: Targeting the ubiquitin-proteasome system to activate wild-type p53 for cancer therapy. Semin Cancer Biol 20: 29-39, 2010.

9. Yu ZK, Gervais JL and Zhang H: Human CUL-1 associates with the SKP1/SKP2 complex and regulates p21(CIP1/WAF1) and cyclin D proteins. Proc Natl Acad Sci USA 95: 11324-11329, 1998.

10. Ciechanover A: The 2008 Lindau Nobel Laureate Meeting: Aaron Ciechanover, Chemistry 2004. J Vis Exp 29: 1559, 2009.

11. Hoeller D and Dikic I: Targeting the ubiquitin system in cancer therapy. Nature 458: 438-444, 2009.

12. Hochstrasser M: Origin and function of ubiquitin-like proteins. Nature 458: 422-429, 2009.

13. Bhoj VG and Chen ZJ: Ubiquitylation in innate and adaptive immunity. Nature 458: 430-437, 2009.

14. Hirsch C, Gauss R, Horn SC, Neuber O and Sommer T: The ubiquitylation machinery of the endoplasmic reticulum. Nature 458: 453-460, 2009.

15. Bergink $S$ and Jentsch S: Principles of ubiquitin and SUMO modifications in DNA repair. Nature 458: 461-467, 2009.

16. Raiborg C and Stenmark H: The ESCRT machinery in endosomal sorting of ubiquitylated membrane proteins. Nature 458: 445-452, 2009.

17. Paoluzzi L and O'Connor OA: Mechanistic rationale and clinical evidence for the efficacy of proteasome inhibitors against indolent and mantle cell lymphomas. BioDrugs 20: 13-23, 2006.

18. O'Connor OA: Marked clinical activity of the proteasome inhibitor bortezomib in patients with follicular and mantle-cell lymphoma. Clin Lymphoma Myeloma 6: 191-199, 2005.

19. Yang Y, Kitagaki J, Dai RM, Tsai YC, Lorick KL, Ludwig RL, Pierre SA, Jensen JP, Davydov IV, Oberoi P, et al: Inhibitors of ubiquitin-activating enzyme (E1), a new class of potential cancer therapeutics. Cancer Res 67: 9472-9481, 2007.

20. Soucy TA, Smith PG and Rolfe M: Targeting NEDD8-activated cullin-RING ligases for the treatment of cancer. Clin Cancer Res 15: 3912-3916, 2009. 
21. Soucy TA, Smith PG, Milhollen MA, Berger AJ, Gavin JM, Adhikari S, Brownell JE, Burke KE, Cardin DP, Critchley S, et al: An inhibitor of NEDD8-activating enzyme as a new approach to treat cancer. Nature 458: 732-736, 2009.

22. Brownell JE, Sintchak MD, Gavin JM, Liao H, Bruzzese FJ, Bump NJ, Soucy TA, Milhollen MA, Yang X, Burkhardt AL, et al: Substrate-assisted inhibition of ubiquitin-like protein-activating enzymes: The NEDD8 E1 inhibitor MLN4924 forms a NEDD8-AMP mimetic in situ. Mol Cell 37: 102-111, 2010.

23. Lin JJ, Milhollen MA, Smith PG, Narayanan U and Dutta A: NEDD8-targeting drug MLN4924 elicits DNA rereplication by stabilizing Cdt1 in S phase, triggering checkpoint activation, apoptosis, and senescence in cancer cells. Cancer Res 70: 10310-10320, 2010.

24. Rabut $G$ and Peter M: Function and regulation of protein neddylation. 'Protein modifications: Beyond the usual suspects' review series. EMBO Rep 9: 969-976, 2008.

25. Leonardi GC, Candido S, Falzone L, Spandidos DA and Libra M: Cutaneous melanoma and the immunotherapy revolution (Review). Int J Oncol 57: 609-618, 2020. (Review).

26. Kuryk L, Bertinato L, Staniszewska M, Pancer K, Wieczorek M, Salmaso S, Caliceti P and Garofalo M: From conventional therapies to immunotherapy: Melanoma treatment in review. Cancers (Basel) 12: 3057, 2020.

27. Tanda ET, Vanni I, Boutros A, Andreotti V, Bruno W, Ghiorzo P and Spagnolo F: Current state of target treatment in BRAF mutated melanoma. Front Mol Biosci 7: 154, 2020.

28. Qiu Y, Zheng Y, Wu KP and Schulman BA: Insights into links between autophagy and the ubiquitin system from the structure of LC3B bound to the LIR motif from the E3 ligase NEDD4 Protein Sci 26: 1674-1680, 2017

29. Huang DT, Ayrault O, Hunt HW, Taherbhoy AM, Duda DM, Scott DC, Borg LA, Neale G, Murray PJ, Roussel MF, et al: E2-RING expansion of the NEDD8 cascade confers specificity to cullin modification. Mol Cell 33: 483-495, 2009.

30. Butt G, Yaylim I, Attar R, Aras A, Romero MA, Qureshi MZ, Purenovic J and Farooqi AA: NEDD4 family of E3 ubiquitin ligases in breast cancer: Spotlight on SMURFs, WWPs and NEDD4. Adv Exp Med Biol 1152: 365-375, 2019.

31. Wang ZW, Hu X, Ye M, Lin M, Chu M and Shen X: NEDD4 E3 ligase: Functions and mechanism in human cancer. Semin Cancer Biol 67: 92-101, 2020.

32. Zhang Y, Qian H, Wu B, You S, Wu S, Lu S, Wang $P$, Cao L, Zhang $\mathrm{N}$ and Sun Y: E3 Ubiquitin ligase NEDD4 family-regulatory network in cardiovascular disease. Int J Biol Sci 16: 2727-2740, 2020.

33. Soysouvanh F, Giuliano S, Habel N, El-Hachem N, Pisibon C, Bertolotto C and Ballotti R: An Update on the role of ubiquitination in melanoma development and therapies. J Clin Med 10: 1133, 2021.
34. Xie P, Peng Z, Chen Y, Li H, Du M, Tan Y, Zhang X, Lu Z, Cui CP, Liu CH, et al: Neddylation of PTEN regulates its nuclear import and promotes tumor development. Cell Res 31: 291-311, 2021.

35. Guo Y, Yang L, Lei S, Tan W and Long J: NEDD4 negatively regulates GITR via ubiquitination in immune microenvironment of melanoma. OncoTargets Ther 12: 10629-10637, 2019.

36. Huang X, Chen J, Cao W, Yang L, Chen Q, He J, Yi Q, Huang H, Zhang $\mathrm{E}$ and Cai Z: The many substrates and functions of NEDD4-1. Cell Death Dis 10: 904, 2019.

37. Livak KJ and Schmittgen TD: Analysis of relative gene expression data using real-time quantitative PCR and the 2(-Delta Delta C(T)) Method. Methods 25: 402-408, 2001.

38. Zou X, Levy-Cohen G and Blank M: Molecular functions of NEDD4 E3 ubiquitin ligases in cancer. Biochim Biophys Acta 1856: 91-106, 2015.

39. Zhang X, Zhang YL, Qiu G, Pian L, Guo L, Cao H, Liu J, Zhao Y, Li X, Xu Z, et al: Hepatic neddylation targets and stabilizes electron transfer flavoproteins to facilitate fatty acid $\beta$-oxidation. Proc Natl Acad Sci USA 117: 2473-2483, 2020.

40. Luhtala S, Staff S, Kallioniemi A, Tanner M and Isola J: Clinicopathological and prognostic correlations of HER3 expression and its degradation regulators, NEDD4-1 and NRDP1, in primary breast cancer. BMC Cancer 18: 1045, 2018.

41. Shao G, Wang R, Sun A, Wei J, Peng K, Dai Q, Yang W and Lin Q: The E3 ubiquitin ligase NEDD4 mediates cell migration signaling of EGFR in lung cancer cells. Mol Cancer 17: 24, 2018.

42. Wen W, Li J, Wang L, Xing Y, Li X, Ruan H, Xi X, Xiong J and Kuang R: Inhibition of NEDD4 inhibits cell growth and invasion and induces cell apoptosis in bladder cancer cells. Cell Cycle 16: 1509-1514, 2017.

43. Meurette O: Shaping of the tumor microenvironment by Notch signaling. Adv Exp Med Biol 1223: 1-16, 2020.

44. Rubey M, Chhabra NF, Gradinger D, Sanz-Moreno A, Lickert H, Przemeck GKH and Hrabě de Angelis M: DLL1- and DLL4-mediated Notch signaling is essential for adult pancreatic islet homeostasis. Diabetes 69: 915-926, 2020.

45. Fleming T, Balderas-Márquez JE, Epardo D, Ávila-Mendoza J, Carranza $M$, Luna $M$, Harvey $S$, Arámburo $C$ and Martínez-Moreno CG: Growth hormone neuroprotection against kainate excitotoxicity in the retina is mediated by Notch/PTEN/Akt signaling. Invest Ophthalmol Vis Sci 60: 4532-4547, 2019

46. Zheng D, Tao M, Liang X, Li Y, Jin J and He Q: p66Shc regulates podocyte autophagy in high glucose environment through the Notch-PTEN-PI3K/Akt/mTOR pathway. Histol Histopathol 35: 405-415, 2020.

This work is licensed under a Creative Commons Attribution 4.0 International (CC BY-NC 4.0) License 